TP Periodica Polytechnica Mechanical Engineering

https://doi.org/10.3311/PPme.12183

Creative Commons Attribution (i)

RESEARCH ARTICLE

\section{Effects of Injection Molding Screw Tips on Polymer Mixing}

\author{
Dániel Török ${ }^{1}$, József Gábor Kovács ${ }^{1 *}$
}

Received 05 March 2018; accepted after revision 04 May 2018

\begin{abstract}
In all fields of industry it is important to produce parts with good quality. Injection molded parts usually have to meet strict requirements technically and aesthetically. The aim of the measurements presented in our paper is to investigate the aesthetic appearance, such as surface color homogeneity, of injection molded parts. It depends on several factors, the raw material, the colorants, the injection molding machine and the processing parameters. In this project we investigated the effects of the injection molding machine on surface color homogeneity. We focused on injection molding screw tips and investigated five screw tips with different geometries. We produced flat specimens colored with a masterbatch and investigated color homogeneity. To evaluate the color homogeneity of the specimens, we used digital image analysis software developed by us. After that we measured the plastication rate and the melt temperature of the polymer melt because mixing depends on these factors. Our results showed that the screw tips (dynamic mixers) can improve surface color homogeneity but they cause an increase in melt temperature and a decrease in the plastication rate.
\end{abstract}

\section{Keywords}

injection molding, color homogeneity, dynamic mixer, process parameters, melt mixing

\footnotetext{
${ }^{1}$ Department of Polymer Engineering, Faculty of Mechanical Engineering, Budapest University of Techology and Economics, H-1521 Budapest, P.O.B. 91, Hungary

*Corresponding author, e-mail: kovacs@pt.bme.hu
}

\section{Introduction}

The objective of this project is to study the appearance of injection molded plastic products and investigate the dispersion of colorants in the polymer melt during the injection molding process improved by screw tips (dynamic mixers). It is a crucial issue because plastic parts are produced and raw materials and colorants are used in an increasing amount worldwide. It is essential to achieve correct and even colour during the manufacturing process to make marketable products. It is important not only in the case of injection molding but in the case of biomaterials [1], 3D printing [2-3], composites [4] and extrusion, too. The quality of color homogeneity depends on several factors, such as the chemical composition of the raw material and colorants, the processing parameters and the machine as well.

In the polymer industry colorants fall into two groups, pigments and dyes. Pigments are colorants which do not dissolve in the polymer matrix, while dyes go into solution [5]. Pigments are classified as organic and inorganic. Their effects on surface color homogeneity was examined by Zsíros et al. [6]. All the three types of colorants have their advantages and disadvantages. Inorganic pigments have better thermal stability but are dispersed worse than organic pigments or dyes. Zsíros et al. [7] have investigated the effects of the composition (carrier, colorants and additives) of masterbatches on surface color homogeneity.

In injection molding the basic and most important mixing equipment is the reciprocating screw [8], but its mixing efficiency is limited and in most cases it is not adequate. To achieve a more homogenous mixture, different types of mixing equipment can be used, such as static and dynamic mixers, which can be installed onto the injection unit or into the screw itself [9]. Static mixers can be fixed onto the injection unit on the front of the screw and consist of a combination of identical elements which are turned $90^{\circ}$ relative to the neighbouring elements. The polymer melt flows through these elements and the mixer splits, reorients and recombines the melt flow. They have good mixing efficiency, but they cause a significant pressure drop. A lot of static mixers with different geometries can be used to mix the polymer melt. Thakur et al. [10] summarized static mixers and their field of applications. In injection molding the Kenics 
(Chemineer) and SMX (Koch Engineering) type static mixers are widely used. Rauline et al. [11] have compared these two static mixers in their paper using numerical simulation.

To improve the mixing efficiency of the injection unit, a special screw design can be used instead of the general purpose screw. Dynamic mixing sections, such as the Maddock mixing section, mixing pins, or cavity transfer mixers [12] can be inserted into the screw body [13]. There are some non-return valve designs available on the market which can improve the mixing efficiency of the injection molding screw [14-15].

In our project we investigated the mixing efficiency of screw tips via digital image processing. Image processing techniques are widely used not only in the polymer industry, but in microstructural analysis [16], geology [17], food engineering [18], and in other fields as well. There are several methods to quantify the degree of mixing. Daumann et al. [19] investigated the mixing process in a discontinuously operated powder mixer with a Matlab®-based image analyser method. Aissa et al. [20] studied the mixing of multicomponent polymer powder blends and the process parameters of the rotating cylinder mixer. These methods can be used to evaluate not only powder mixtures but also the surface color homogeneity of injection molded parts.

L. Zsíros et al. [21] investigated methods to evaluate homogeneity and defined an algorithm capable of quantifying the inhomogeneity of injection molded samples. First, the color coordinates of the pixels of a scanned picture are converted into the Lab color space from RGB. After this, a moving window (Fig. 1) with a side width of 35 pixels scans the picture and the mean color coordinates are calculated in every position. In each position, the Euclidian distances of the color values of the pixel and the mean color value were calculated according to Eq. (1).

$$
\Delta E=\sqrt{\Delta L^{2}+\Delta a^{2}+\Delta b^{2}}
$$

where $\Delta \mathrm{E}$ is the color difference, $\Delta \mathrm{L}$ is the difference of the average lightness of the pixel and the image, $\Delta \mathrm{a}$ is the average "a" value of the pixel and the image, while $\Delta \mathrm{b}$ is the average "b" value of the pixel and the image.

The standard deviation of these distances can characterize the evenness of color inside the window. The image is characterized with the highest mean standard deviation (HMD). In order to correlate with human perception better, we modified the HMD values (Eq. (2)):

$$
I H=5 \cdot[\log (H M D-G M D)+1]
$$

where $I H$ is the level of inhomogeneity, $H M D$ is the highest mean standard deviation and $G M D$ is the standard deviation of Euclidean distances that belong to the window size of 610 pixels (image size: 610x610 pixel). In this work, this measuring method was used to calculate homogeneity.

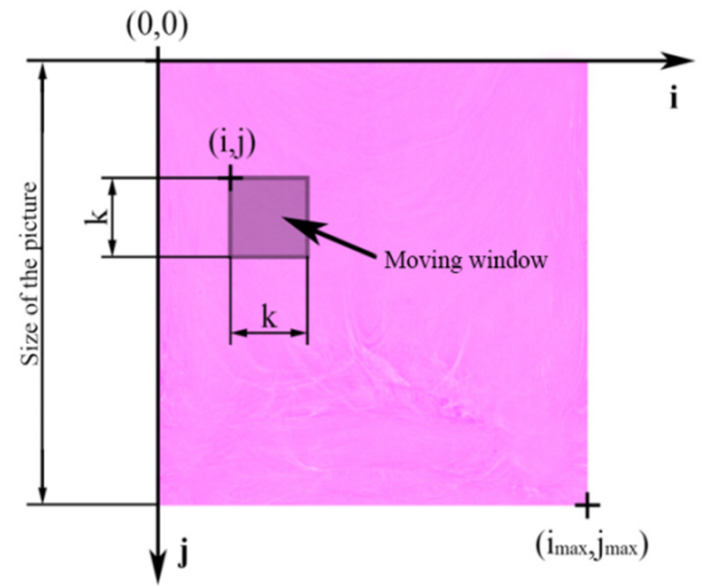

Fig. 1 Digitized image of a test specimen and the measuring method with the moving window

\section{Machines, materials and methods}

We investigated five different dynamic mixers. Each of them was mounted onto the screw as a non-return valve (Fig. 2). Dynamic mixer type screw tips consist of two parts: the rotor, which is attached to the rotating screw, and the mixing ring, which does not turn with the screw and the rotor. For each mixer, we used the smallest flow cross section to characterize its geometry. These gaps - the smallest flow cross section (Fig. 2) - were 14, 38, 56, 91 and $211 \mathrm{~mm}^{2}$. The dynamic mixers were manufactured with conventional technology and with Direct Metal Laser Sintering, which is already used to manufacture mold inserts [22].

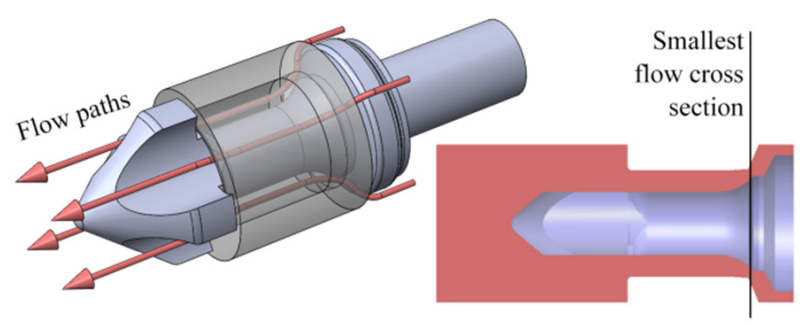

Fig. 2 Illustration of the flow path in a typical dynamic mixer and the smallest flow cross section

The test specimens were injection molded on an Arburg Allrounder Advance 270S 400-170 machine. The injection unit of the injection molding machine has a universal single flight, three-zone nitrided screw of a diameter of $30 \mathrm{~mm}$ and an L/D ratio of 20. Its maximum axial displacement is $120 \mathrm{~mm}$, therefore the maximum shot volume is $85 \mathrm{~cm}^{3}$. The highest value of the injection pressure and holding pressure was 2000 bar. The screw is position-regulated, therefore the shot volume set in the injection molding parameters can be maintained more accurately during manufacturing. 
The specimens were made in a two-cavity mold, which produces $80 \times 80 \mathrm{~mm}$ flat specimens with a thickness of $1.2 \mathrm{~mm}$. The cavity of the mold is polished in order to minimize the surface roughness of the specimens as the smooth surface increases the accuracy of color inhomogeneity measurement. The injection molding parameters are summarized in Table 1. The specimens were produced from unfilled acrylonitrile-butadiene-styrene (ABS, Terluran GP-35 from Styrolution Group $\mathrm{GmbH}$ ) with $4 \mathrm{wt} \%$ of masterbatch (MB, Renol-pink ABS143479Q from Clariant).

Table 1 The injection molding parameters

\begin{tabular}{lc}
\hline Injection molding parameter & Value \\
\hline Volume $\left[\mathrm{cm}^{3}\right]$ & 26 \\
Injection rate $\left[\mathrm{cm}^{3} / \mathrm{s}\right]$ & 55 \\
Switchover volume $\left[\mathrm{cm}^{3}\right]$ & 5 \\
Holding pressure $[\mathrm{bar}]$ & 600 \\
Holding time [s] & 6 \\
Residual cooling time $[\mathrm{s}]$ & 11 \\
Screw rotational speed $[\mathrm{m} / \mathrm{min}]$ & 10,25 and 50 \\
Backpressure $[\mathrm{bar}]$ & 60 \\
Decompression volume $\left[\mathrm{cm}^{3}\right]$ & 6 \\
Decompression rate $\left[\mathrm{cm}^{3} / \mathrm{s}\right]$ & 20 \\
Barrel temperature at nozzle $\left[{ }^{\circ} \mathrm{C}\right]$ & 225 \\
Mold temperature $\left[{ }^{\circ} \mathrm{C}\right]$ & 40 \\
\hline
\end{tabular}

The injection molded parts were digitized with a flatbed scanner (Epson Perfection V600 Photo). The plastification rate was measured according to the EUROMAP 5 standards on the Arburg Allrounder Advance 270S 700-290 machine. The melt temperature was measured with a Fluke 51 II type single input digital thermometer. The accuracy of the equipment is $0.05 \%+0.4^{\circ} \mathrm{C}$.

500 specimens were injection molded with each dynamic mixer. All relevant process parameters (machine cycle number, cycle time, maximum injection pressure, switchover pressure, switchover volume, cushion and screw rotation time) were recorded on the injection molding machine for each cycle.

The plastification rate was determined for three different screw rotation speeds $(10,25$ and $50 \mathrm{~m} / \mathrm{min})$ with 5 independent measurements for each. The melt temperature was measured five times during purging. The melt temperature was measured for 20-30 seconds in the purged material for each measurement, then the highest temperature values were averaged and used as the melt temperature for the given process parameter set.

We used an algorithm based on the standard deviation method to evaluate the images because the flow lines and color inhomogeneity can be detected quite precisely. Finally, the test specimens were digitized with a flatbed scanner for the homogeneity measurements and the digital images were evaluated with our own algorithm described earlier in the introduction [21].
The image analyzer software characterizes the unevenness of color in the pictures (Fig. 1).

\section{Results and discussion}

In injection molding one of the most important process parameters is melt temperature. The lower the viscosity is, the better the flow properties are, and therefore the better the mold cavity is filled. On the other hand, too high a melt temperature can cause the degradation of the polymer molecules. Dynamic mixers can raise the melt temperature during plasticization because of their small flow cross section. The laminar flow of the polymer melt through the screw tip increases inner friction in the melt and this produces extra heat. As a result, the temperature of the melt increases to a greater extent than in the case of screw tips with a large cross section. The melt temperature rise was examined as a function of the flow cross section of dynamic mixers (Fig. 3). Temperature rise follows a power function of the smallest flow cross section, at the screw rotation speed of $25 \mathrm{~m} / \mathrm{min}$, used in the experiments. As is known, the ABS melt cannot flow through a smaller gap than $0.05 \mathrm{~mm}$, therefore this is the smallest theoretical gap size. The smallest flow cross section calculated from this gap size is about $4.7 \mathrm{~mm}^{2}$, which gives a maximum temperature rise of about $82^{\circ} \mathrm{C}$. Melt temperature cannot exceed the maximum processing temperature allowed for the material, and processing must fulfil several other criteria, too.

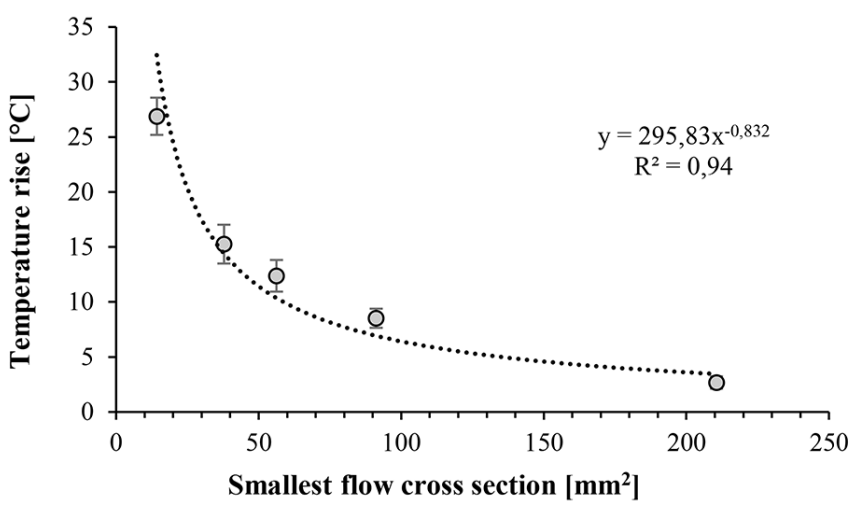

Fig. 3 Temperature rise in the melt as a function of the gap size in the mixers, with a screw rotation speed of $25 \mathrm{~m} / \mathrm{min}$

For the measurements of plastification rate, screw rotation speed was fixed at 10, 25 and $50 \mathrm{~m} / \mathrm{min}$ (Fig. 4). It can be stated that dynamic mixers act as flow resistance, which highly depends on screw rotation speed. For each screw rotation speed the plastification rate has a limit, which is the maximum throughput of the injection screw without the screw tip. The plastification rate increases as the smallest flow cross section increases.

The throughput of the given screw and screw tip can be described with Eq. (3), where $\operatorname{Pr}\left(v_{\text {screw }}\right)$ is the throughput, $v_{\text {screw }}$ is screw rotation speed, $A$ is the flow cross section and $c$ is a screw type-dependent constant. We used the saturation curve as 
a basis for creating the curve. The equation is derived from the measured data; the curve was fitted to the measured points. The maximum throughput for a given screw is in a linear relationship with the screw rotation speed.

$$
\operatorname{Pr}\left(v_{\text {screw }}\right)=c \cdot v_{\text {screw }} \cdot \sqrt{1-e^{\sqrt{\frac{A}{\sqrt{v_{\text {screw }}}}}}}
$$

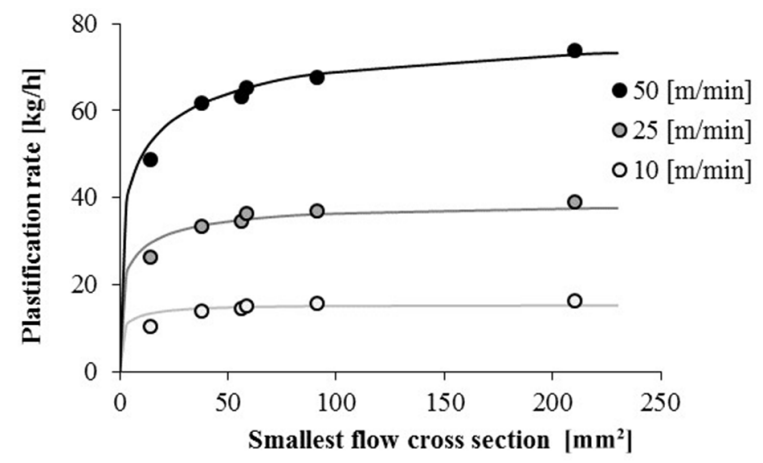

Fig. 4 Plastification rate as a function of the geometry and screw rotation speed of the dynamic mixer. (The dots are the measured values, the lines are the calculated functions.)

The maximum injection pressures decreased as the smallest flow cross section decreased (Fig. 5). This phenomenon is related to the shear that develops in dynamic mixers, and thus the temperature change of the polymer melt. As melt temperature increases, the viscosity of the melt drops, thus filling pressure decreases as well. This allows longer flow paths, and the shrinkage and warpage of the products can be controlled better. Thanks to the better fluidity of the melt, the compensation of the decrease of specific volume can also be more effective. The effect of the screw tip on the polymer has to be taken into account because small cross-section screw tips strain the plastic more and can lead to degradation.

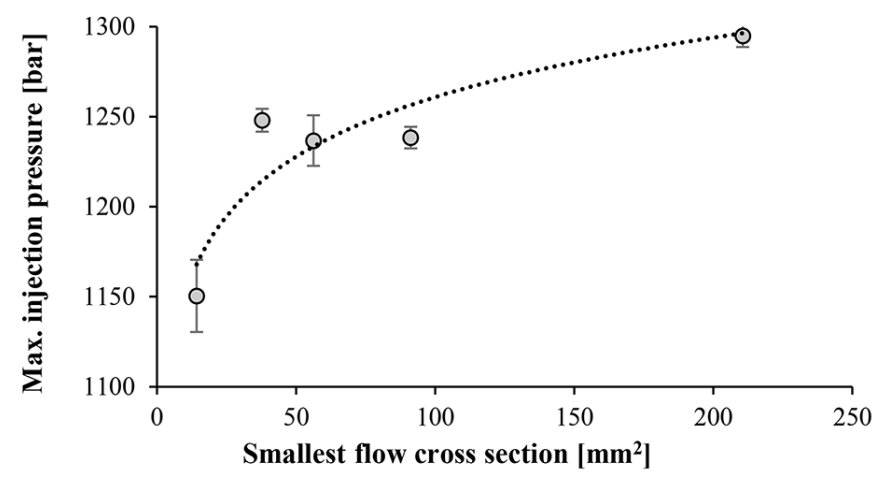

Fig. 5 Injection pressure as a function of the smallest flow cross section.

Homogeneity is directly proportional to the shear rate and melt temperature. The effect of melt temperature on homogeneity was measured and the results show that inhomogeneity increases as melt temperature increases (Fig. 6). As the viscosity of the polymer melt decreases, homogeneity is reduced because less shear is produced and this results in worse mixing.

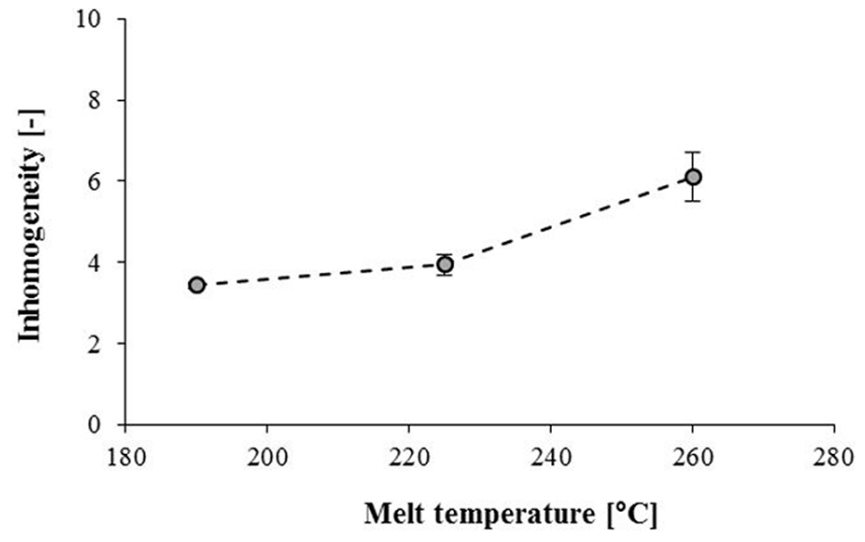

Fig. 6 The homogeneity of the samples molded with different melt temperatures

An increase in the shear rate has the opposite effect. The higher the shear rate, the better homogeneity is. The effect of shear rate on mixing was investigated by Kasaliwal et al. [23]. They used polycarbonate filled with multi-walled carbon nanotubes (MWCNT). They also showed that as mixing performance (shear rate) increases, the MWCNT agglomerates get smaller, therefore mixing gets better. The resultant of the effects of shear rate and melt temperature probably determines inhomogeneity, as we illustrated in Fig. 7.

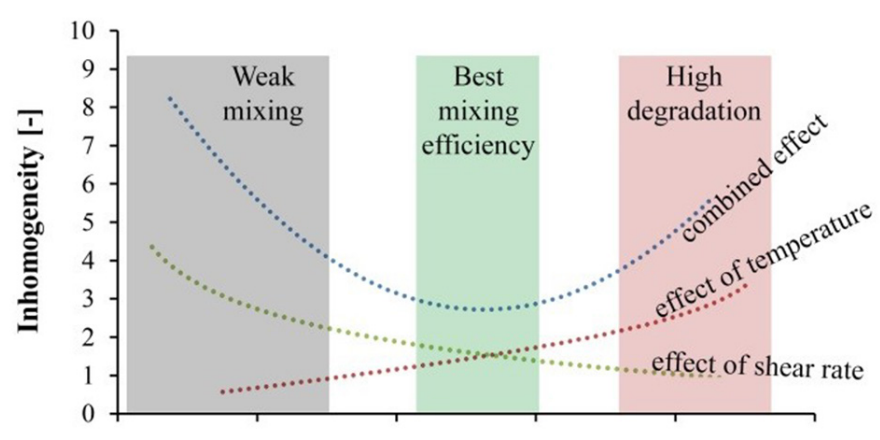

Shear rate / Melt temperature

Fig. 7 The homogeneity of the samples molded with different melt temperatures

We evaluated the homogenization ability of dynamic mixers. Fig. 8 shows the homogeneity of the samples as a function of gap size. Gap size has a direct relationship with shear rate and the increase in temperature. Similarly to Fig. 7, as gap size decreases, homogeneity first improves up to a certain limit, then it gets worse. Therefore homogeneity has an optimum, where the quality of the samples is the best.

Decreasing gap size in the higher ranges results in better quality. The reason for this is increasing shear rate, which leads to better mixing. In this range shearing does not increase melt temperature considerably. In the range of significantly smaller gap sizes increasing shear rate results in a considerable increase in temperature, which causes worse homogeneity as a result of a change in viscosity. 


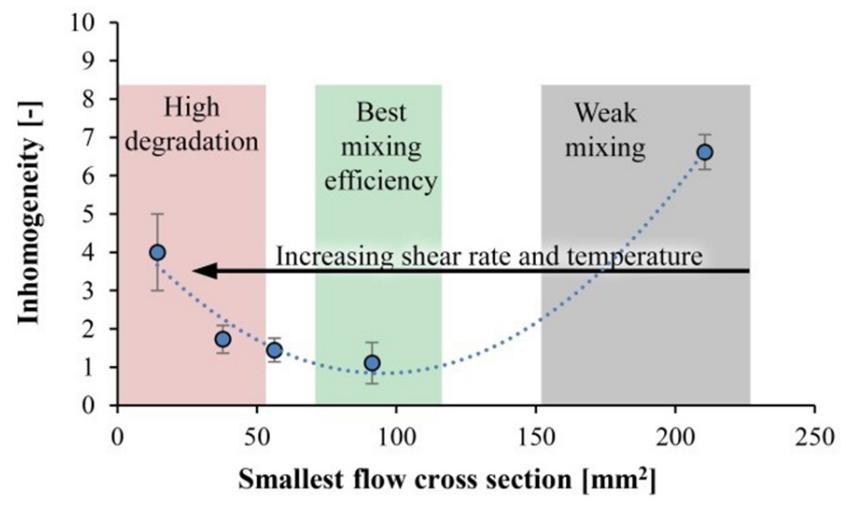

Fig. 8 The homogeneity of the samples as a function of the smallest flow cross section.

\section{Conclusion}

Our goal was to characterize different dynamic mixers based on their smallest flow cross section. We investigated the effects of the gap size of dynamic mixers on the process parameters of injection molding, the temperature of the polymer melt, and the homogeneity of the samples. We measured the actual temperature of the melt and proved that melt temperature increases more at small cross-section screw tips due to higher shear rates. In the case of the smallest cross section this increase reached $27^{\circ} \mathrm{C}$. This increase in temperature affects injection pressure. Hotter, more fluid melt fills the cavity better, therefore injection pressure can decrease by up to 150 bar. Measurements showed that the efficiency of mixing depends on the shear rate during plastification and on the actual temperature of the melt. It was also proved that higher melt temperatures decrease color homogeneity, whereas a higher shear rate increases color homogeneity. Small cross-section screw tips cause a greater increase in temperature and higher shear rates, whereas in the case of higher cross-section screw tips both the increase in temperature and the shear rate are lower. The efficiency of mixing is determined by the resultant of the two opposite effects. We showed that the efficiency of mixing as a function of flow cross section has an optimum in the investigated parameter range.

\section{Acknowledgement}

The authors wish to thank Arburg Hungária Kft. for the Arburg Allrounder 370S 700-290 injection molding machine, Lenzkes GmbH for the clamping tool system, and Tool-Temp Hungary Kft. and Piovan Hungary Kft. for their support.

\section{References}

[1] Bocz, K., Tábi, T., Vadas, D., Sauceau, M., Fages, J., Marosi, Gy. "Characterisation of natural fibre reinforced PLA foams prepared by supercritical CO2 assisted extrusion." eXPRESS Polymers Letters. 10(9), pp. 771-779. 2017.

https://doi.org/10.3144/expresspolymlett.2016.71
[2] Rahim, T. N. A. T., Abdullah, A. M., Md Akil, H., Mohamad, D., Rajion, Z. A. "The improvement of mechanical and thermal properties of polyamide 12 3D printed parts by fused deposition modelling." eXPRESS Polymers Letters. 11(12), pp. 963-982. 2017.

https://doi.org/10.3144/expresspolymlett.2017.92

[3] Rochlitz, B., Pammer, D. "Design and analysis of 3D printable foot proesthesis." Periodica Polytechnica Mechanical Engineering. 61(4), pp. 282-287. 2017.

https://doi.org/10.3311/PPme.11085

[4] Subramani, M., Arumugam, A. B., Ramamoorthy, M. "Vibration Analysis of Carbon Fiber Reinforced Laminated Composite Skin with Glass Honeycomb Sandwich Beam Using HSDT." Periodica Polytechnica Mechanical Engineering. 61(3), pp. 213-224. 2017. https://doi.org/10.3311/PPme.9747

[5] Harris, R. M. "Coloring technology for plastics." Plastics Design Library, New York. 1999.

[6] Zsíros, L., Török D., Kovács J. G. "Evaluation of the homogenization properties of masterbatches." Coloration Technology. 133(5), pp. 431438. 2017.

https://doi.org/10.1111/cote. 12298

[7] Zsíros, L., Török, D., Kovács, J. G. "The Effect of Masterbatch Recipes on the Homogenization Properties of Injection Molded Parts." International Journal of Polymer Science. 2017, pp. 1-7. 2017. https://doi.org/10.1155/2017/5745878

[8] Schwartz, H W. "Reciprocating screw injection molding machine." US3335461 A, USA, 1964.

[9] Rauwendaal, C. J. "Screw extruder with improved dispersive mixing." US5932159, USA, 1999.

[10] Thakur, R. K., Vial, Ch., Nigam, K. D. P., Nauman, E. B., Djelveh, G. "Static mixers in the process industries - a review." Chemical Engineering Research and Design. 81(7), pp. 787-826. 2003. https://doi.org/10.1205/026387603322302968

[11] Rauline, D., Le Ble, A Vec J.-M., Bousquet, J., Tanguy, P. A. "A Comparative Assessment of the Performance of the Kenics and SMX Static Mixers." Chemical Engineering Research and Design. 78(3), pp. 389-396. 2000. https://doi.org/10.1205/026387600527284

[12] Rauwendaal, C. "Polymer mixing: Self-study guide." Carl Hanser Verlag, Munich. 1998

[13] Rauwendaal, C. J. "Screw extruder with improved dispersive mixing." US5932159, USA, 1999.

[14] Hindmarch, R. S. "The Cavity Transfer Mixer: A Blender for all Seasonings." Materials \& Design. 8(6), pp. 331-339. 1987. https://doi.org/10.1016/0261-3069(87)90083-5

[15] Rauwendaal, C., Osswald, T., Gramann, P., Davis, B. "A New Dispersive Mixer for Single Screw Extruders." In: 56th SPE ANTEC, Atalanta, Georgia, pp. 277-283. 1998

[16] Collins, P. C., Welk, B., Searles, B., Tiley, J., Russ, J. C., Fraser, H. L. "Development of methods for the quantification of microstructural features in $\alpha+\beta$-processed $\alpha / \beta$ titanium alloys." Materials Science and Engineering A. 508, pp. 174-182. 2009. https://doi.org/10.1016/j.msea.2008.12.038

[17] Zhang, Q., Skjetne, R. "Image processing for identification of sea-ice floes and the floe size distributions." IEEE Transactions on Geoscience and Remote Sensing. 53(5), pp. 2913-2924. 2015. https://doi.org/10.1109/TGRS.2014.2366640

[18] Elmasry, G. M., Nakauchi, S. "Image analysis operations applied to hyperspectral images for non-invasive sensing of food quality - A comprehensive review." Biosystems Engineering. 142, pp. 53-82. 2016. https://doi.org/10.1016/j.biosystemseng.2015.11.009 
[19] Daumann, B., Fath A., Anlauf H., Nirschl H. "Determination of the mixing time in a discontinuous powder mixer by using image analysis." Chemical Engineering Science. 64, pp. 2320-2331. 2009.

https://doi.org/10.1016/j.ces.2009.01.032

[20] Aissa, A. A., Duchesne, C., Rodrigue, D. "Polymer powders mixing part II: Multi-component mixing dynamics using RGB color analysis." Chemical Engineering Science. 65(12), pp. 3729-3738. 2010. https://doi.org/10.1016/j.ces.2010.03.007

[21] Zsíros, L., Suplicz, A., Romhány, G., Tábi, T., Kovács, J. G. "Development of a novel color inhomogeneity test method for injection molded parts." Polymer Testing. 37, pp. 112-116. 2014.

https://doi.org/10.1016/j.polymertesting.2014.05.009
[22] Zink, B., Szabó, F., Hatos, I., Suplicz, A., Kovács, N. K., Hargitai, H., Tábi, T., Kovács, J. G. "Enhanced Injection Molding Simulation of Advanced Injection Molds." Polymers. 9(2), pp. 1-11. 2017. https://doi.org/10.3390/polym9020077

[23] Kasaliwal, G. R., Pegel, S., Göldel, A., Pötschke, P., Heinrich, G. "Analysis of agglomerate dispersion mechanisms of multiwalled carbon nanotubes during melt mixing in polycarbonate." Polymer. 51, pp. 27082720. 2010.

https://doi.org/10.1016/j.polymer.2010.02.048 